Ivonne Buhlmann · Edmund Wascher

\title{
Intentional pre-cueing does not influence the Simon effect
}

Received: 21 January 2004/ Accepted: 4 August 2004/Published online: 25 January 2005

(C) Springer-Verlag 2005

\begin{abstract}
Choice reactions can be performed more quickly if the response corresponds spatially to the stimulus, even when the stimulus location is irrelevant for the task (Simon effect). It is assumed that the Simon effect is related to interference between spatial stimulus and response codes in a response selection stage. A central finding for such a response selection account is the increase in the effect if the most probable response location is given in advance by an intentional pre-cue. However, Hasbroucq and Possamaï (1994) assumed that the increase in the Simon effect in such a task may be due to an unmeant pre-cueing of the stimulus location, which has been recently supported by an electroencephalography (EEG) study by Wascher and Wolber (2004). In the present study this notion has been tested experimentally. In Experiment 1, a centrally presented symbolic cue served as an intentional cue. As a result, the enhancement of the Simon effect in valid cueing almost disappeared. When tactile cues were used (Experiment 2), the increase in the Simon effect disappeared completely. Thus, the influence of intentional cueing reported in previous studies can be assigned to attentional factors and does not support a response selection account.
\end{abstract}

\section{Introduction}

Choice reactions can be performed more quickly and accurately if the response corresponds spatially to the stimulus, even when the stimulus location is irrelevant to the task. That effect of the irrelevant stimulus location on RT has come to be known as the Simon effect (see

I. Buhlmann $(\bowtie) \cdot$ E. Wascher

Max Planck Institute for Human Cognitive and Brain Sciences, Amalienstrasse 33, 80799 Munich, Germany

E-mail: Buhlmann@cbs.mpg.de also Simon, 1969; Simon \& Small, 1969; Simon, Craft, \& Wester, 1973; Simon \& Rudell, 1967). It is assumed that the Simon effect is due to the interference between differing spatial codes within a response selection stage. To select the correct response, the nonspatial code of the stimulus (its identity) must be translated into a response code. This process is assumed to be influenced by an automatically generated response code that derives from the task-irrelevant stimulus position. Response selection is slowed when the two spatial codes indicate different response locations, but is accelerated when they indicate the same location.

Evidence of such a response selection account comes from two sources. Firstly, from experiments that tested stimulus-response (S-R) correspondence in simple response time (RT) tasks, and secondly, from experiments that used intentional pre-cueing.

In simple response tasks, where the task does not require the selection of one among several response alternatives, no S-R correspondence effects were found (Berlucchi, Crea, Di Stefano, \& Tassinari, 1977; Anzola, Bertoloni, Buchtel, \& Rizzolatti, 1977). However, Hommel (1996) also reported a Simon effect for simple responses whenever a simple response has been occasionally followed by an alternative response. Thus, not the response selection itself but the fact that a response alternative was available appeared to be the crucial factor in obtaining a Simon effect.

The second stream of evidence of the response selection account of the Simon effect derives from studies that used intentional pre-cueing. In this kind of task, the response required for the forthcoming trial is pre-cued at a particular level of validity. Consistently, it has been reported that the Simon effect increases with valid intentional pre-cueing compared with a regular, uncued task (Proctor, Lu, \& Van Zandt, 1992; Proctor \& Wang, 1997; Verfaellie, Bowers, \& Heilman, 1988; Wascher \& Wolber, 2004).

The first to report results of a pre-cueing task were Verfaellie et al. (1988). They used as target stimuli brightly or dimly filled circles, on the left or right of a 
fixation cross. The targets were preceded by two pre-cue stimuli, one above and the other below the fixation cross. The upper pre-cue indicated the probable response and the lower pre-cue showed the most probable stimulus location. The cues were a picture of an index finger pointing to the left or right position. They were presented in the middle vertical line of the screen with a validity of $80 \%$. In every trial, stimulus location, response location, both, or neither of them could be pre-cued. Verfaellie and colleagues (1988) found an increased Simon effect by a pre-cue that signaled the likely response. On the other hand, the Simon effect was not affected by a pre-cue that signaled the likely stimulus position.

The effects reported by Verfaellie et al. (1988) could have been influenced by the rather complex information that was delivered by the cue. To provide more stringent tests of whether response pre-cueing enhances the Simon effect, Proctor et al. (1992) used a simpler procedure and manipulated intentional and attentional cues in separate blocks. Also, with this simpler setting, an enhancement of the Simon effect with valid intentional pre-cues and a tendency toward a reversed Simon effect with invalid intentional pre-cueing were reported. Thus, the enhancement of the Simon effect with valid intentional pre-cueing did not depend on the experimental set-up used by Verfaellie et al. (1988; see also Wascher \& Wolber, 2004).

This finding was taken as evidence of the notion that, in Simon tasks, spatial codes interfere within a response selection stage. However, the direction of the modification of the Simon effect is unexpected. If valid intentional cueing allows for advance response selection (Wascher \& Wolber, 2004), this stage should be less affected by interference. Hence, reduced Simon effects would be expected with valid intentional cueing (Buckholz, Odonnell, \& McAuliffe, 1996).

An alternative interpretation of the enhancement of the Simon effect with intentional pre-cueing was proposed by Hasbroucq and Possamaï (1994). In a structural analysis of the experiments of Proctor et al. (1992), they listed all possible relations between spatial codes that are involved in the task. In this analysis it turned out that cue validity and S-R correspondence are not independent of each other. In validly cued trials the cue points toward the stimulus position whenever S-R relations correspond (Fig. 1). In validly cued noncorresponding trials, however, the cue points at the location opposite the target. Thus, the effect of S-R correspondence might be attenuated by an attentional effect that is equally directed. In invalidly cued trials S-R correspondence and attentional effect might counteract and, therefore, this interaction also explains smaller Simon effects with invalid intentional cues (e.g., Proctor et al., 1992; Wascher \& Wolber, 2004; see Fig. 1). Thus, since all studies so far used spatial cues (either pointing fingers or arrows), it is possible that the enhancement of the Simon effect was due to the effects of spatial attention rather than the fact that response-relevant advance information was given.

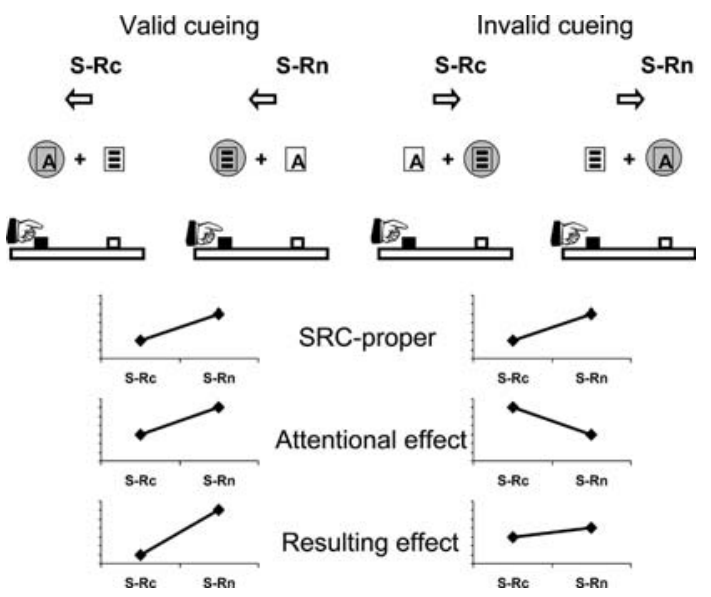

Fig. 1 The possible interaction of spatial parameters in an intentional cueing task (top). If a shift in visuo-spatial attention were evoked by the cue - even though it is intentional - the target would be attended in validly cued stimulus-response (S-R) corresponding $(S-R c)$ and in invalidly cued noncorresponding $(S$ $R n$ ) trials (see gray circles). Possible response time (RT) effects of shifts in visuo-spatial attention are depicted at the bottom. In validly cued trials enhanced effects of S-R correspondence would be expected

Wascher and Wolber (2004) tested this attentional explanation by means of psychophysiological parameters of spatial attention, in particular temporal aspects of a posterior asymmetry in the electroencephalogram (EEG). This phenomenon was initially described as the $\mathrm{N} 2 \mathrm{pc}$ (N2 posterior contralateral; Luck \& Hillyard, 1994a, 1994b) and reflects an increase in cortical negativity at posterior electrode sites contralateral to the location of a task-relevant stimulus. Its peak latency was assumed to be related to the speed at which a stimulus can be localized (Wolber \& Wascher, 2003, 2004). These latency effects have been shown to be independent of an underlying N2 component. Therefore, it will be referred to more neutrally as the posterior contralateral negativity (PCN; Van der Lubbe, Jaskowski, Wauschkuhn, \& Verleger, 2001).

Wascher and Wolber (2004) used a very similar set-up to that introduced by Proctor et al. (1992). A centrally presented directional cue was used that informed about the likely response location. The behavioral measures replicated the results of previous studies. The PCN indicated perceptual acceleration, as proposed by Hasbroucq and Possamaï (1994). The authors found a reduced latency of the PCN peak whenever the target appeared at the side indicated by the cue, even though the cue indicated the most likely response location. The PCN rose $31.5 \mathrm{~ms}$ earlier if the cue pointed toward the location of the upcoming stimulus than if the cue was pointing away from the target location. Thus, it appears plausible that perceptual factors were involved in the modification of the Simon effect by intentional cueing, which is not directly related to mechanisms evoking the Simon effect. Moreover, the lateralized readiness potential (LRP), which is thought to be related to 
selective response activation processes in primary motor areas (Coles, 1989; Gratton, Coles, Sirevaag, Eriksen, \& Donchin, 1988; De Jong, Wierda, Mulder, \& Mulder, 1988), indicated that the main mechanisms involved in the Simon effect remained unaffected both by intentional and by attentional pre-cueing.

This evidence, however, can be criticized as being indirectly obtained from psychophysiological measures. Therefore, we conducted two experiments testing this hypothesis more directly by experimental manipulations of the intentional pre-cue task. In both experiments it was intended to minimize the possible influence of spatial attention evoked by the cue upon the spatial parameters involved in generating the Simon effect.

\section{Experiment 1: Symbolic cueing}

In Experiment 1, a centrally presented symbolic cue with no obvious directional information was used as an intentional pre-cue. All other parameters of the procedure were exactly the same as in the study by Wascher and Wolber (2004). As the explicit directional factor was missing, the perceptual influence should be reduced or eliminated. This should seen in both behavioral and EEG data.

\section{Methods}

\section{Participants}

Seven women and five men aged between 20 and 37 years (average age 27.7 years) were paid volunteers in this experiment. All participants were right-handed and had normal or corrected-to-normal vision. They were in good physical health and had no neurological or psychiatric disorders. All participants took part in all three tasks reported here (details see below; uncued, symbolic cueing, tactile cueing) in counterbalanced order.

\section{Stimuli and procedure}

The participants were seated in a dimly lit, electrically shielded chamber. A 22-inch computer screen was placed approximately $140 \mathrm{~cm}$ in front of them. The stimuli were generated by customized software (graphical card: VSG 2/5 from Cambridge Research Systems). They were presented on a dark background and the participants had to respond on a customized keyboard with their index fingers.

Each trial began with the presentation of an intentional pre-cue (a red or a green circle). The pre-cues were $7 \times 7 \mathrm{~mm}$ (visual angle approximately $.3^{\circ}$ ). They appeared for $200 \mathrm{~ms}$ in the middle of the screen and indicated with a validity of $80 \%$ the required response hand.

With an stimulus onset asynchrony (SOA) of $500 \mathrm{~ms}$ ( $\pm 100 \mathrm{~ms})$, the target stimulus was presented. The letters $A$ or $B$ in bright yellow (14 mm wide and $13 \mathrm{~mm}$ high, visual angle $\left..6^{\circ} \times .5^{\circ}\right)$ appeared in one of two quadratic frames $(20 \times 20 \mathrm{~mm}$, visual angle approximately $.8^{\circ}$ ) and a noise object (three horizontal timbers) appeared inside the other frame. The participants were instructed to respond to the letter $A$ with the left key and to the letter $B$ with the right key. The target stimuli were presented for $200 \mathrm{~ms}$ for a total of 600 trials. All four types of stimuli (left $A$, left $B$, right $A$, right $B$ ) as well as valid and invalid cues, were presented in a randomized order.

The duration of the task was about $25 \mathrm{~min}$. There was one break after 300 trials.

\section{Recording and data processing}

The EEG was recorded from 61 scalp positions using $\mathrm{Ag} / \mathrm{AgCl}$ electrodes (Picker-Schwarzer). $\mathrm{Cz}$ served as reference. The electro-oculogram (EOG) was recorded for the detection of ocular artifacts. Bipolar recordings of the vertical and horizontal EOG were made from electrodes above and below the right eye as well as from electrodes at the outer canthi of both eyes. The electrode impedances were kept below $5 \mathrm{k} \Omega$. EOG and EEG were filtered and amplified using Neuroscan DC amplifiers with a band-pass filter of $0-200 \mathrm{~Hz}$ and digitized at $500 \mathrm{~Hz}$. Trials with artifacts were excluded from further analysis. Transmission of vertical EOG and horizontal EOG into the EEG was subtracted from the EEG data (Gratton, Coles, \& Donchin, 1983).

\section{Data analysis}

Response parameter Response time (RT) is denoted as the time between the appearance of the target stimulus and the response. Trials with incorrect responses or responses faster than 200 or slower than $900 \mathrm{~ms}$ were defined as response errors and excluded from RT and EEG analysis.

The average RTs and proportion of errors of each participant were determined and entered first into an ANOVA with the factors S-R correspondence (2, corresponding vs. noncorresponding trials) and cueing (2, valid cueing vs. uncued task). Note that the comparison of the Simon effect between the uncued and validly cued trials remains the core measure since it reflects the change in the Simon effect due to the cue.

EEG parameter The EEG was averaged separately for each of the four experimental conditions (valid vs. invalid, corresponding vs. noncorresponding trials). Event-related lateralizations (ERLs) were obtained by computing contralateral-ipsilateral difference potentials: EEG activity ipsilateral to the target location was subtracted from activity contralateral to the target, separately for left and right stimuli. Subsequently, these difference potentials were averaged, resulting in a difference wave that shows only stimulus-related 
lateralizations of the EEG with respect to the position of the target. Note that this procedure is basically identical to the method by which the lateralized readiness potential (LRP) is calculated (see Coles, 1989), except that the stimulus location was the relevant spatial code and not the side of response. PCN latencies were determined as the moment of maximal amplitude of ERLs between $180 \mathrm{~ms}$ and $300 \mathrm{~ms}$ after presentation of the stimulus display at parietal-occipital electrodes (PO7, PO8). Peak latencies and amplitudes were also entered into an ANOVA with the factors S-R correspondence (2) and cue validity (2; valid vs. invalid).

To measure response activation evoked by the cue, the lateralized readiness potential (LRP) was calculated time locked to the cue at those electrodes most closely located to hand motor areas $(\mathrm{FC} 3 / \mathrm{FC} 4)$. The mean amplitude of the LRP in the cue-target interval was tested with $t$-tests against zero for four time windows of $50 \mathrm{~ms}$ duration each (beginning at $100 \mathrm{~ms}$ before and ending $100 \mathrm{~ms}$ after the average moment of target onset).

\section{Results}

\section{Response parameter}

Comparing RTs of the regular Simon effect with the Simon effect in validly cued trials (Table 1), a clear acceleration in responses by the cue was observed, $F(1,11)=13.404, p<.001$. Participants responded faster to corresponding than to noncorresponding trials, $F(1,11)=54.687, p=.000$. Crucial to this study was the interaction of cue validity by S-R correspondence, which did not reach significance, $F(1,11)=.511, p>.2$. Only in the direct comparison between validly and invalidly cued trials was an interaction of cue validity by S-R correspondence observed, $F(1,11)=4.999, p=.047$. This interaction relied primarily on a reduction in the Simon effect in invalidly cued trials, $F(1,11)=12.320, p=.005$. No enhancement of the Simon effect was observed for validly cued trials, $F(1,11)=.511, p>2$.

Comparing error rates (see Table 1) for validly cued trials and the uncued task no effect of cueing was observed, $F(1,11)=.451, p>.2$. More errors were committed in noncorresponding than in corresponding trials, $F(1,11)=31.578, p<.001$. Again, the interaction of cueing by S-R correspondence did not reach significance, $F(1,11)=.355, p>.2$. Also, in the comparison of validly and invalidly cued trials this interaction did not reach significance, $F(1,11)=.656, p>.2$.

\section{EEG parameter}

The PCN (Fig. 2) did not change latency due to any experimental manipulation: Cue validity $F(1,11)=.877$, $p>.2$; S-R correspondence $F(1,11)=.021, p>.2$; cue validity by S-R correspondence $F(1,11)=.708, p>.2$. Also, for PCN peak amplitude neither a main effect of cue validity, $F(1,11)=.972, p>.2$, nor a main effect of S-R correspondence, $F(1,11)=.825, p>.2$, was observed. However, PCN peak amplitudes were significantly reduced whenever the cue indicated the location of the upcoming stimulus as being visible in the interaction between cue validity and S-R correspondence, $F(1,11)=8.569, \quad p=.014$. This result might indicate facilitated target localization if the cue appeared in the same location as the prepared response.

A comparison between PCN amplitudes measured in the no cue condition and the valid pre-cue condition (see Fig. 2 right panel) shows a marginal significant interaction effect of cueing by S-R correspondence, $F(1,11)=4.408, p=.060$, but no such interaction effect for invalidly cued trials, $F(1,11)=.739, p=.408$.

The LRP (Fig. 3) indicated response activation during the cue-target interval. Already $100 \mathrm{~ms}$ preceding the moment of average target presentation, the LRP over hand motor areas differed significantly from zero,

Table 1 Mean response time $(R T)$ and error rates for Experiment 1 (symbolic visual cueing) and Experiment 2 (tactile cueing) as a function of the cueing condition. Standard deviations are given in parentheses

\begin{tabular}{llll}
\hline Cueing & S-R corresponding & S-R noncorresponding & Simon effect \\
\hline Experiment 1: Symbolic color cueing & & & $391(64.0)$ \\
$\quad$ Valid mean RT & $360.8(50.3)$ & $5.9(4.1)$ & $30.2(18.7)$ \\
Error \% & $2.5(3.2)$ & $470.4(53.7)$ & $3.4(3.6)$ \\
Invalid mean RT & $454.1(59.5)$ & $24(19.7)$ & $16.3(22)$ \\
Error \% & $19.0(21.6)$ & $389.4(7.9)$ \\
Experiment 2: Tactile cueing & $353.4(49.0)$ & $6.4(5.7)$ & $36(15.0)$ \\
$\quad$ Valid mean RT & $2.7(7.0)$ & $452.7(51.4)$ & $3.7(3.8)$ \\
Error \% & $420.4(48.2)$ & $17.9(21.0)$ & $32.3(18.6)$ \\
Invalid mean RT & $12.5(18.0)$ & $4.4(4.4)$ \\
Error \% & $406.1(38.8)$ & $5.7(2.5)$ & $34.2(17.0)$ \\
Control & $1.6(1.2)$ & $4.1(.7)$ \\
$\quad$ No cueing mean RT & & \\
Error \% & & & \\
\hline
\end{tabular}

Since the study was an intraindividual design, the data were checked for the influence of the sequence of tasks performed. No significant influence was observed on any measure 


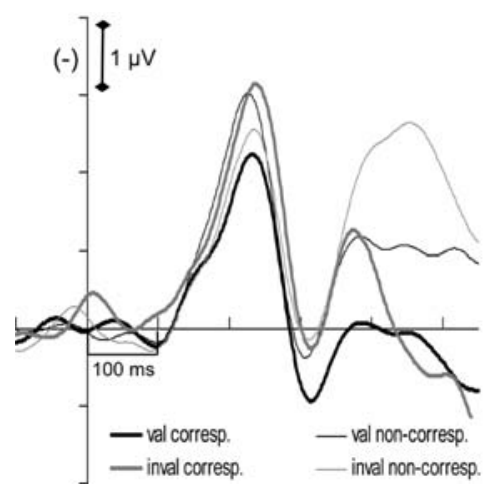

Fig. 2 Posterior contralateral negativity (PCN) effects in Experiment 1 depicted from target onset until $550 \mathrm{~ms}$. In the left panel validly and invalidly cued trials are superposed. In the right panel, data from validly cued and uncued trials are superposed. It is evident from both plots that the amplitude of PCN was reduced

$t(11)=3.160, p=.009$. Also, within the three successive intervals the LRP remained reliable above zero: -50 to $0 \mathrm{~ms} t(11)=2.905, p=.014 ; 0$ to $50 \mathrm{~ms} t(11)=3.611$, $p=.004 ; 50$ to $100 \mathrm{~ms} t(11)=3.470, p=.005$.

\section{Discussion}

Experiment 1 was conducted to test the influence of a symbolic nonspatial intentional pre-cue upon the Simon effect. In contrast to previous studies that used directional cues (Verfaellie et al., 1988; Proctor et al., 1992; Proctor \& Wang, 1997; Wascher \& Wolber, 2004) the Simon effect did not increase with valid intentional precues. Only when validly and invalidly cued trials were compared directly, a small interaction between cue validity and $\mathrm{S}-\mathrm{R}$ correspondence was observed. In the present study the Simon effect appeared to be reduced with invalid intentional pre-cues rather than enhanced with valid cues. This lack of consistency across studies using intentional pre-cueing indicates that the increase in

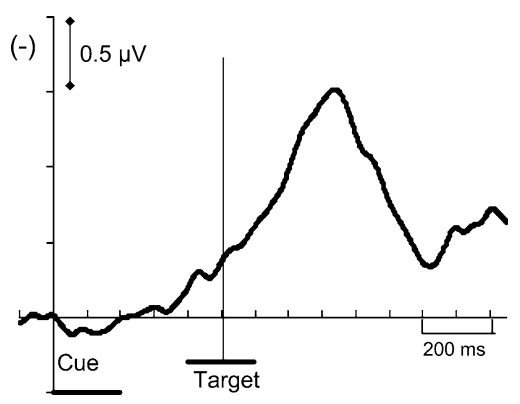

Fig. 3 Lateralized readiness potential (LRP) as an indicator of response preparation in the cue-target interval in Experiment 1. Since neither cue validity nor S-R correspondence can be defined based on the cue direction alone, data from all conditions were collapsed. The deviation of the LRP from the baseline shows that participants obviously prepared the response that was indicated by the cue

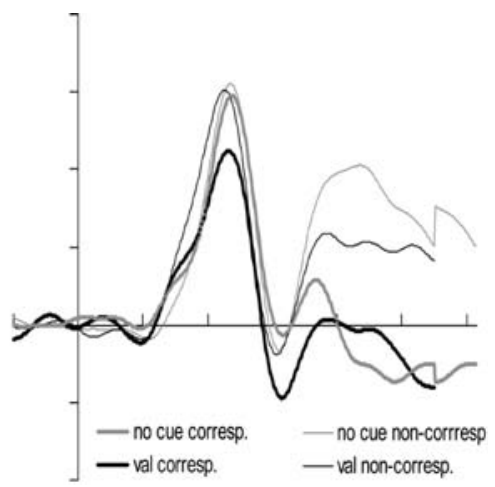

whenever the target appeared in a spatial location that corresponded to the side given as the most probable response location (validly cued-S-R corresponding; invalidly cued-S-R noncorresponding)

the Simon effect with intentional pre-cueing may not be a direct consequence of response preparation based on cue information. Alternatively, the modulation of the Simon effect might be due to attentional factors (Hasbroucq \& Possamaï, 1994). The cues, indicating the most probable response location, additionally point to a particular location in space. Whenever the target stimulus appears in that location, its processing might be accelerated. This account had been supported recently by an EEG study (Wascher \& Wolber, 2004) that showed faster posterior EEG asymmetries whenever the pre-cued response location corresponded to the position of the upcoming target stimulus. The present study shows similar results. Although no clear differences in the latency of the PCN were observed, the reduction in the PCN amplitude might correlate with an attentional shift toward the side of the most probable response. The lack of a latency effect might be due to the reduced saliency of the spatial properties of the cue, which is in line with the behavioral effects. However, residual attentional effects might be reflected in changes in PCN amplitude. It was selectively reduced whenever the indicated response side corresponded to the target location.

Therefore, the reduction in the spatial information of the cue, by using nonspatial symbolic cues, appears adequate to reduce the influence of spatial attention upon the magnitude of the Simon effect. However, the cue used was not sufficient to eliminate this influence completely.

We claim here that reduced but still evident influence of spatial attention could be responsible for this finding. However, although the LRP clearly indicated response activation in the cue-target interval, and although responses were accelerated by valid cues, it might also be assumed that the influence of the nonspatial cue upon the motor system might have been weaker than the influence of directional cues used previously. Also, in that case, reduced influence of intentional cueing on the Simon effect would have been expected. 


\section{Experiment 2: Tactile cueing}

Thus, in the following experiment, a type of cueing was used that delivered no visual information but explicit tactile advice about the required response side.

If visuo-spatial attention is the origin of the enhanced Simon effect with valid pre-cues as reported in previous studies (e.g., Wascher \& Wolber, 2004), no effect of intentional cueing would be expected with tactile cues. In contrast, if the intensity of information delivered by the cue is responsible, the reduction in the Simon effect with valid intentional pre-cueing should be evident, as with symbolic spatial pre-cues, or even increased.

In Experiment 2, a tactile vibration of the palms served as an intentional pre-cue. This type of pre-cueing should affect only response selection and not the distribution of visuo-spatial attention.

\section{Methods}

\section{Participants}

The same participants took part in this experiment as in Experiment 1.

\section{Stimuli and procedure}

Most aspects of the methods used for this experiment were similar to those reported for Experiment 1. However, in this experiment the response pre-cue was not a colored circle but a tactile vibration of the palm of either hand. The vibrations were produced by two "Thera Tapper" pulsers, which were implemented in the response board. The pulsers were located approximately $80 \mathrm{~mm}$ away from the response keys. Vibrations with a duration of $200 \mathrm{~ms}$ were applied to the palm of either the left or the right hand, indicating which hand will have to respond to the target with a validity of $80 \%$.

\section{Recording and data processing}

Recording and data processing were performed in the same manner as in Experiment 1.

\section{Data analysis}

The data analysis was identical to Experiment 1.

Results

\section{Response parameters}

When comparing validly cued trials with the uncued Simon effect, a reliable effect of S-R correspondence was observed, $F(1,11)=90.129, p<.001$. Additionally, the cue clearly accelerated responses, $F(1,11)=15.205$, $p=.002$. Quantitatively, the effect of the tactile cue $(52 \mathrm{~ms})$ was larger than the effect of the symbolic color cue in Experiment 1 (47 ms). However, again, the effect of the S-R correspondence did not interact with the validity of the cues, $F(1,11)=.106, p>.2$ (see Table 1 ). Therefore, as in Experiment 1, the Simon effect did not increase with valid intentional cueing. The absolute difference between the Simon effect obtained with validly cued trials and the uncued Simon effect was smaller than $2 \mathrm{~ms}$ (36.0 vs. $34.2 \mathrm{~ms})$ and therefore even numerically negligible. Even when validly and invalidly cued trials were compared directly, the interaction of cue validity by $\mathrm{S}-\mathrm{R}$ correspondence did not reach significance, $F(1,11)=.442, p>.2$.

For error percentages (see Table 1), the effect of S-R correspondence reached significance for the comparison of validly cued trials and the uncued block, $F(1,11)=34.367, p<.001$. As is known in the case of Simon tasks, more errors were committed with noncorresponding trials. No effect of cueing, $F(1,11)=.729$, $p>.2$, and no interaction of $\mathrm{S}-\mathrm{R}$ correspondence by cueing, $F(1,11)=.053, p>.2$, were observed. Only when validly and invalidly cued trials were compared a significant main effect of cue validity, $F(1,11)=5.205$, $p=.043$, indicated an increase in errors with invalid cueing. This effect, however, did also not interact with $\mathrm{S}-\mathrm{R}$ correspondence, $F(1,11)=.857, p>.2$.

\section{EEG parameter}

As in Experiment 1, the ERLs were calculated over the parietal occipital electrode pair PO7/PO8. The PCN (Fig. 4) peaked slightly (by $2 \mathrm{~ms}$ ) later in corresponding than in noncorresponding trials, $F(1,11)=5.664$, $p=.037$. A marginal interaction of S-R correspondence by cue validity, $F(1,11)=4.452, p=.059$, was due to an increase in the latter effect in validly cued trials but a slight reversal in invalidly cued trials. However, note that those effects are not related to behavior. Also, for the peak amplitude of the PCN a marginal interaction of S-R correspondence by cue validity was observed, $F(1,11)=4.462, p=.058$. This effect was due to larger amplitudes whenever the direction indicated by the cue coincided with the location of the upcoming target stimulus. Neither of the main effects reached significance: Cue validity $F(1,11)=2.175, p=.168$; S-R correspondence $F(1,11)=.218, p>.2$.

Note that neither of the effects for the PCN indicated a visual attentional shift in the direction of the cued response. The effects observed are instead a consequence of the huge tactile EEG asymmetries evoked by the cue, which are still visible at the moment of stimulus presentation (see Fig. 4). Since such tactile asymmetries are assumed to be inverted in polarity (Wascher \& Wauschkuhn, 1996), most probably due to surfaceto-depth current flow in pyramidal tract neurons in the somatosensory cortex (Lang, Höllinger, Eghker, \& Lindinger, 1994), their influence led to reversed effects in 


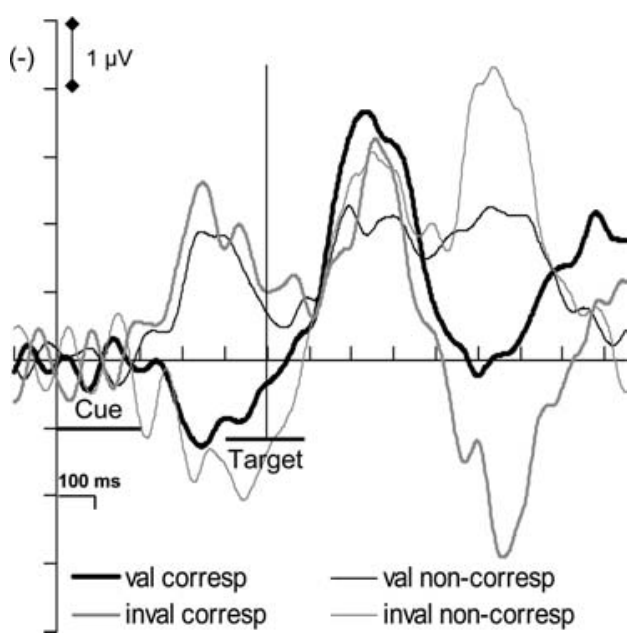

Fig. 4 PCN effects in Experiment 2. Since tactile stimuli have been assumed to evoke posterior electroencephalogram (EEG) asymmetries (Lang et al. 1994), the event-related lateralizations of the EEG are depicted from cue onset until $550 \mathrm{~ms}$ after target onset. It is obvious that the target event-related lateralizations (ERLs) might be still influenced by the cue ERLs. Nevertheless, no signs of an attentional shift due to the cue direction have been observed

the target PCN, which cannot account for the behavioral effects.

As in Experiment 1, the LRP (Fig. 5) differed reliably from zero in all four time windows around the average moment of target presentation: -100 to $-50 \mathrm{~ms}$ $t(11)=5.298, \quad p<.001 ; \quad-50$ to $0 \mathrm{~ms} \quad t(11)=4.565$, $p=.001 ; 0$ to $50 \mathrm{~ms} t(11)=4.595, p=.001 ; 50$ to $100 \mathrm{~ms}$ $t(11)=5.711, p<.001$. Thus, also in Experiment 2 clear evidence of response preparation in the cue-target interval was found.

\section{Discussion}

By the use of salient tactile cues, the increase in the Simon effect with valid intentional cueing was not restored. Although valid tactile cues had a strong effect on RTs, the Simon effect remained the same compared

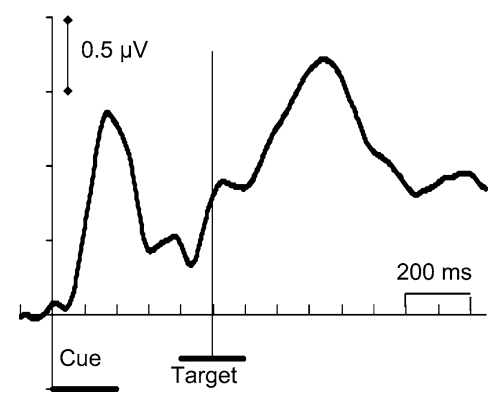

Fig. 5 LRP as an indicator of response preparation in the cuetarget interval in Experiment 2. As for Experiment 1, response preparation is visible preceding the presentation of the target. Additionally, a strong early phasic activation shows the immediate impact of the tactile stimulation upon the motor system with a regular, uncued Simon effect. Thus, these data provide strong evidence of the notion that the increase in the Simon effect with intentional pre-cueing, as reported previously (Proctor et al., 1992; Verfaellie et al., 1988; Wascher \& Wolber, 2004), is a consequence of shifts of visuo-spatial attention rather than of altered response selection processes. According to the notion that tactile cues did not evoke an attentional shift toward the cued location, the psychophysiological parameters did not vary depending on the correspondence between cued response and stimulus location. The marginal effects observed for PCN latency and PCN amplitude are most probably due to the overlap from the tactile cue potential and are not predictive of behavior.

\section{General discussion}

Two experiments were conducted to investigate the impact of intentional pre-cueing with no explicit visuospatial information upon the Simon effect. The increase in the Simon effect with valid intentional cueing has been previously taken as evidence of a response selection account of the Simon effect (Proctor et al., 1992). This notion has been criticized for two reasons. Firstly, if response selection were the locus where the interference between the irrelevant spatial code of the target stimulus and the relevant response code - as delivered by its identity - takes place, intentional cueing should reduce the effect rather than enhance it (Buckholz et al., 1996). Secondly, the use of visuo-spatial cues, namely arrows, might have evoked visual attentional shifts toward the cued location. According to this logic (see also Fig. 1), an overlapping attentional shift evoked by the direction of a symbolic spatial cue should lead to an increase in the Simon effect with valid intentional cueing. Also, a decrease in the Simon effect with invalid intentional cueing should occur. This pattern of results has been observed in a number of previous studies (Proctor et al., 1992; Wascher \& Wolber, 2004). The possible influence of visuo-spatial attention upon the Simon effect with intentional cueing has been recently supported by an ERP study that used the common visuo-spatial type of cues (Wascher \& Wolber, 2004). To deal with the critique that the psychophysiological data provided indirect evidence only, we tested the role of visuo-spatial attention in the modification of the Simon effect with intentional cueing by eliminating visuo-spatial information from the cues. If the attentional interpretation is true, the absence of visuo-spatial information in the cue should eliminate the modification of the Simon effect. On the other hand, if intentional pre-cueing affects the interference of codes in a response selection stage, the modification of the Simon effect should be a function of the intensity of intentional cues.

In Experiment 1, we used a symbolic pre-cue with no explicit visuo-spatial information. The most probable side of the required response was indicated by one 
of two colors. Posterior asymmetries of the EEG showed only minimal effects of attentional shifts toward the cued location. Also, behaviorally, the increase in the Simon effect, as usually observed with visuo-spatial pre-cues, was eliminated. A slight reduction in the Simon effect with invalid cues might have been due to the fact that the pre-cue was still presented visually. Translation of the color into a visuo-spatial code might have constituted the remaining effect. However, it might be argued that the color cue was less salient than the previously used symbolic spatial arrows. Thus, the lack of an increase in the Simon effect might have been due to reduced impact of the cue upon behavior. Hence, in a second experiment, no visual cues were used. Response location was indicated by tactile stimulation. Here, shifts of visuo-spatial attention should not take place and, therefore, the influence of this factor on the Simon effect should disappear. According to this notion, ERP correlates of visuo-spatial attention did not vary with the overlap between the indicated response side and the location of the target stimulus. In line with the attentional account, no modification of the Simon effect was observed in this task.

These results demonstrate that the type of the intentional pre-cue is responsible for the modification of the Simon effect that has been reported previously (Proctor et al., 1992; Verfaellie et al., 1988; Wascher \& Wolber, 2004). As claimed theoretically (Hasbroucq \& Possamaï, 1994), and indicated by a psychophysiological study (Wascher \& Wolber, 2004), directional visual cues (such as arrows) evoke a spatial attentional shift, although the cue is defined as being intentional. As a consequence of this attentional shift, stimuli in the cued location are processed faster. Due to the overlap of spatial codes in such a task, this attentional influence might substantially modify the Simon effect. When this attentional influence is eliminated by using non visuo-spatial cues, the Simon effect is completely unaffected by intentional pre-cueing. Therefore, modifications of the Simon effect in the wellknown intentional cueing task (Proctor et al., 1992; Verfaellie et al., 1988; Wascher \& Wolber, 2004) do not prove the claim that the interference of codes that constitutes the Simon effect takes place in a response selection stage. However, although the results presented here query the central proof of the response selection account of the Simon effect, they do not contradict such an approach.

\section{References}

Anzola, G. P., Bertoloni, G., Buchtel, H. A., \& Rizzolatti, G. (1977). Spatial compatibility and anatomical factors in simple and choice reaction time. Neuropsychologia, 15, 295-302.

Berlucchi, G., Crea, F., Di Stefano, M., \& Tassinari, G. (1977). Influence of spatial stimulus-response compatibility on reaction time of ipsilateral and contralateral hand to lateralized light stimuli. Journal of Experimental Psychology: Human Perception and Performance, 3, 505-517.
Buckolz, E., Odonnell, C., \& McAuliffe, J. (1996). The Simon effect: evidence of a response processing "functional locus". Human Movement Science, 15, 543-564.

Coles, M. G. H. (1989). Modern mind-brain reading: Psychophysiology, physiology, and cognition. Psychophysiology, 26, 251-269.

De Jong, R., Wierda, M., Mulder, G., \& Mulder, L. J. M. (1988). Use of partial stimulus information in response processing. Journal of Experimental Psychology: Human Perception and Performance, 14, 682-692.

Gratton. G., Coles, M. G. H., Donchin, E. (1983). A new method for off-line removal of ocular artifact. Electroencephalography and Clinical Neuropsychology, 55, 468-484.

Gratton, G., Coles, M. G. H., Sirevaag, E. J., Eriksen, C. W., \& Donchin, E. (1988). Pre- and poststimulus activation of response channels: A psychophysiological analysis. Journal of Experimental Psychology: Human Perception and Performance, $14,331-344$

Hasbroucq, T., \& Possamaï, C. A. (1994). What can a precue enhance - an analysis of the experiments of Proctor, Lu and van Zandt (1992). Acta Psychologica, 85, 235-244.

Hommel, B. (1996). S-R compatibility effects without response uncertainty. The Quarterly Journal of Experimental Psychology, 49 A, 546-571.

Lang, W., Höllinger, P., Eghker, A., \& Lindinger, G. (1994). Functional localization of motor processes in the primary and supplementary motor areas. Journal of Clinical Neurophysiology, 11, 397-419.

Luck, S. J., \& Hillyard, S. A. (1994a). Electrophysiological correlates of feature analysis during visual search. Psychophysiology, 31, 291-308.

Luck, S. J., \& Hillyard, S. A. (1994b). Spatial filtering during visual search: Evidence from human electrophysiology. Journal of Experimental Psychology: Human Perception and Performance, 20, 1000-1014.

Proctor, R. W., \& Wang, H. (1997). Enhancement the Simon effect by response-location precues: Evaluation of the stimulus-evaluation account. Acta Psychologica, 95, 279-298.

Proctor, R. W., Lu, C., \& Van Zandt, T. (1992). Enhancement of the Simon effect by response precuing. Acta Psychologica, 81, 53-74.

Simon, J. R. (1969). Reactions towards the source of stimulation. Journal of Experimental Psychology, 81, 174-176.

Simon, J. R., \& Rudell, A. (1967). Auditory S-R compatibility: The effect of an irrelevant cue on information processing. Journal of Applied Psychology, 51, 300-304.

Simon, J. R., \& Small, A. M. (1969). Processing auditory irrelevant information: Interference from an irrelevant cue. Journal of Applied Psychology, 53, 433-435.

Simon, J. R., Craft, J. L., \& Webster, J. B. (1973). Reactions toward the stimulus source: Analysis of correct responses and errors over a five- day period. Journal of Experimental Psychology, 101, 175-179.

Van der Lubbe, R. H. J., Jaskowski, P., Wauschkuhn, B., Verleger, R. (2001). Influence of time pressure in a simple response task, a choice-by-location task, and the Simon task. Journal of Psychophysiology, 15, 241-255.

Verfaellie, M., Bowers, D., \& Heilman, K. M. (1988). Attentional factors in the occurrence of stimulus-response compatibility effects. Neuropsychologia, 26, 435-444.

Wascher, E., \& Wauschkuhn, B. (1996). The interaction of stimulus- and response-related processes measured by event-related lateralizations of the EEG. Electroencephalography and Clinical Neurophysiology, 99, 149-162.

Wascher, E., \& Wolber, M. (2004). Attentional and intentional cueing in a Simon task: An EEG-based approach. Psychological Research, 68, 18-30.

Wolber, M., \& Wascher, E. (2003). Visual search strategies are indexed by event-related lateralizations of the EEG. Biological Psychology, 63, 79-100.

Wolber, M., \& Wascher, E. (2004). Cortical correlates of visuospatial processing. Manuscript submitted for publication. 